Supporting Information for:

\title{
The Impact of Excess Lead Iodide on the Recombination Kinetics in Metal Halide Perovskites
}

Aboma Merdasa ${ }^{l,}{ }^{*}$, Alexander Kiligaridis ${ }^{2}$, Carolin Rehermann ${ }^{l}$, Mojtaba Abdi-Jalebi $^{3}$, Jonas Stöber ${ }^{2}$, Boris $^{2}$ Louis $^{2}$, Marina Gerhard ${ }^{2}$,Samuel D. Stranks ${ }^{3}$,Eva L. Unger ${ }^{1,2, *}$, and Ivan G. Scheblykin, ${ }^{2, *}$

${ }^{1}$ Young Investigator Group Hybrid Materials Formation and Scaling, Helmholtz-Zentrum Berlin für Materialen und Energie GmbH, Albert-Einstein Strasse 16, 12489 Berlin, Germany.

${ }^{2}$ Chemical Physics and NanoLund, Lund University, PO Box 118, 22100 Lund, Sweden.

${ }^{3}$ Cavendish Laboratory, Department of Physics, University of Cambridge, JJ Thomson Avenue, Cambridge CB3 OHE, United Kingdom. 


\section{SI Note 1. Sample Preparation \& Characterization}

We prepared $\mathrm{MAPbI}_{3}$ films in two different ways. For all samples, the "dip" in the PLQY spectrum was observed. Below we give a brief description of the sample preparation methods.

\section{$\mathrm{MAPbI}_{3}$ from DMF (method 1)}

All materials were purchased from Sigma-Aldrich, TCI or Greatcell Solar Ltd and were used as received. 1M solution of $\mathrm{MAI}: \mathrm{PbI}_{2}$ in a ratio of 1:1 in pure $\mathrm{N}, \mathrm{N}$-dimethylformamide (DMF) was prepared from the two stock solutions. Then the solution was spin-coated on top of a clean cover slip glass substrate at $2000 \mathrm{rpm}$ for 60 seconds (accel. of $2000 \mathrm{rpm} \mathrm{s}^{-1}$ ) and annealed at $100^{\circ} \mathrm{C}$ for $15 \mathrm{~min}$. On some of these films, an additional $\mathrm{PbI}_{2}$ layer was thermally evaporated. All preparations were made inside a $\mathrm{N}_{2}$ glovebox. Figure S1a shows the cross-section SEM of the $\mathrm{MAPbI}_{3}$ film where the thickness is estimated to about $325 \mathrm{~nm}$. Figure $\mathrm{S} 1 \mathrm{~b}$ shows the XRD measurements of a $\mathrm{MAPbI}_{3}$ film with and without an evaporated layer of $\mathrm{PbI}_{2}$

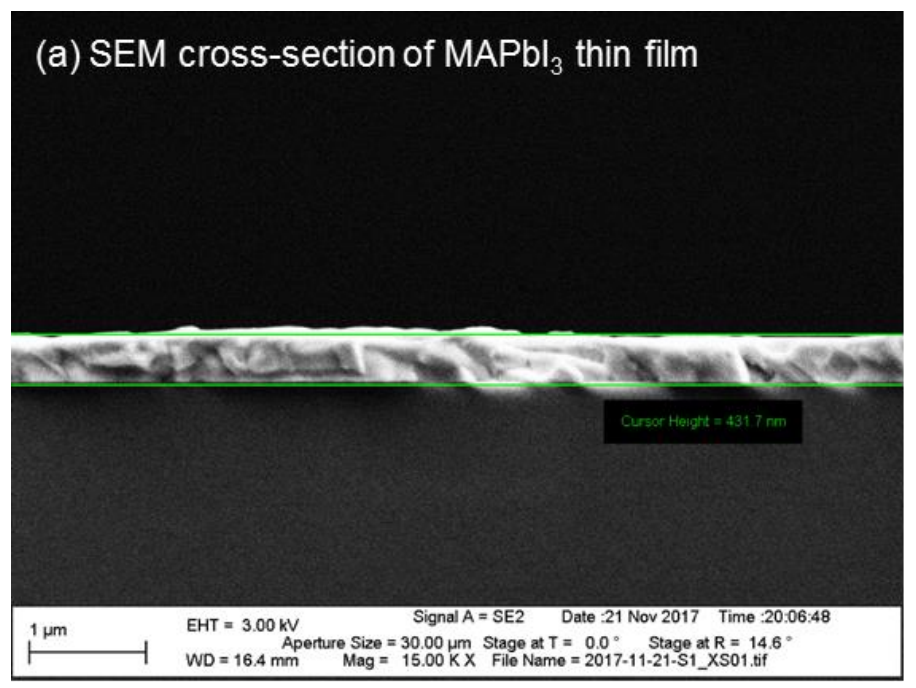

(b) XRD diffractogram of films $\mathrm{w} /$ and $w / 0 \mathrm{Pbl}_{2}$

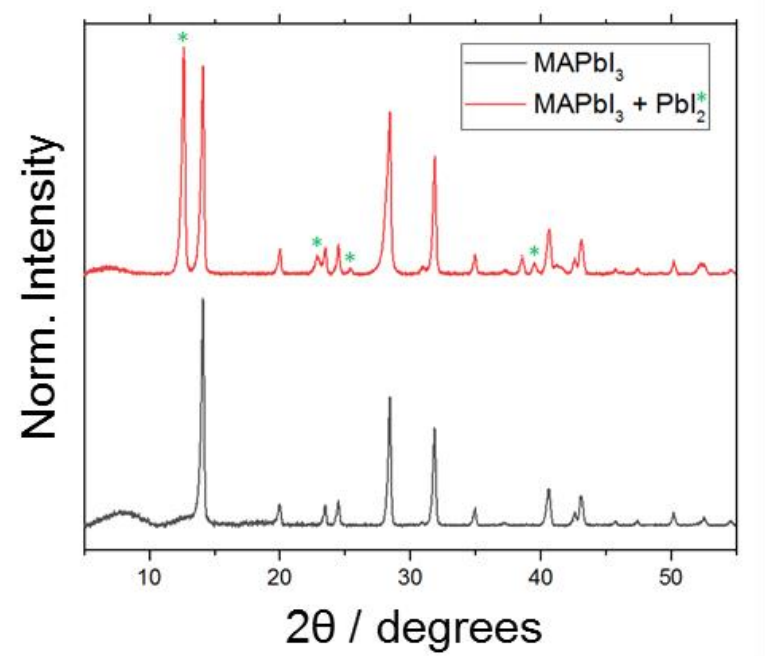

Figure S1. (a) SEM cross section of $\mathrm{MAPbI}_{3}$ film having thickness of $430 \mathrm{~nm}$. (b) XRD diffractograms of $\mathrm{MAPbI}_{3}$ films with a layer of $\mathrm{PbI}_{2}$ (red) and without $\mathrm{PbI}_{2}$ (black). $\mathrm{PbI}_{2}$ peaks are marked with green stars. 


\section{$\mathrm{MAPbI}_{3}$ from DMF (method 2)}

All materials were purchased from Sigma-Aldrich or Acros Organics and were used as received. A 37\% weight solution of $\mathrm{Pb}(\mathrm{Ac})_{2}$ and MAI having a molar ratio of 1:3 were prepared in DMF. The solution was then spin coated on top of a clean cover slip glass substrate at $2000 \mathrm{rpm}$ for 60 seconds (accel. of $2000 \mathrm{rpm} \mathrm{s}^{-1}$ ) and annealed at 100 ${ }^{\circ} \mathrm{C}$ for 15 min to achieve laterally uniform perovskite films.

PLE and absorptance spectra of these films are shown in Figure S2 in the range between $450 \mathrm{~nm}-700 \mathrm{~nm}$ demonstrating the presence of the "dip" after light soaking.

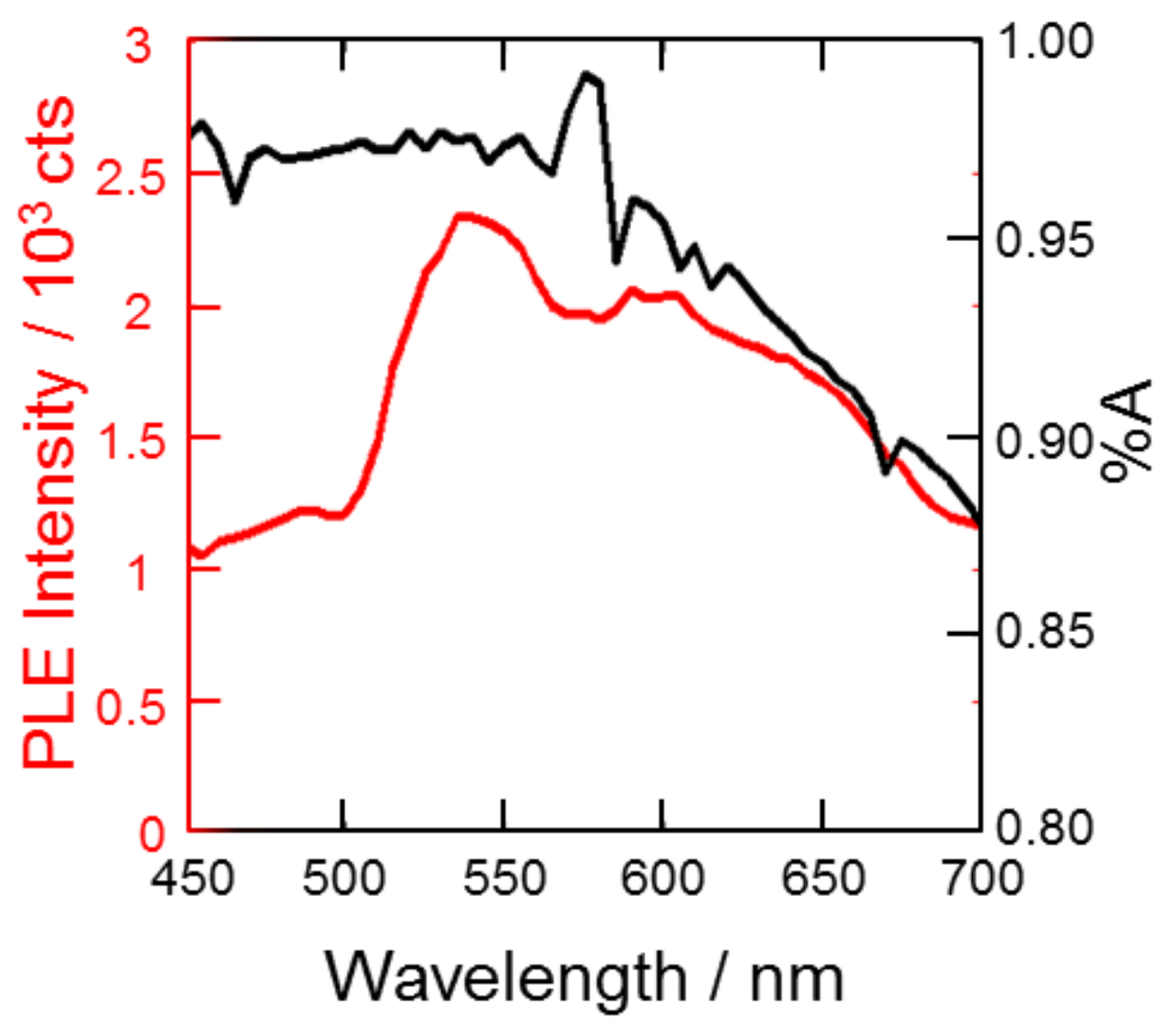

Figure S2. PLE (red) and absorptance (black) spectra from $\mathrm{MAPbI}_{3}$ sample prepared according to the alternative method (method 2). 


\section{SI Note 2. Description of Photoluminescence Excitation Microscopy}

Figure S3 shows a schematic overview of the PL excitation/emission microscope.

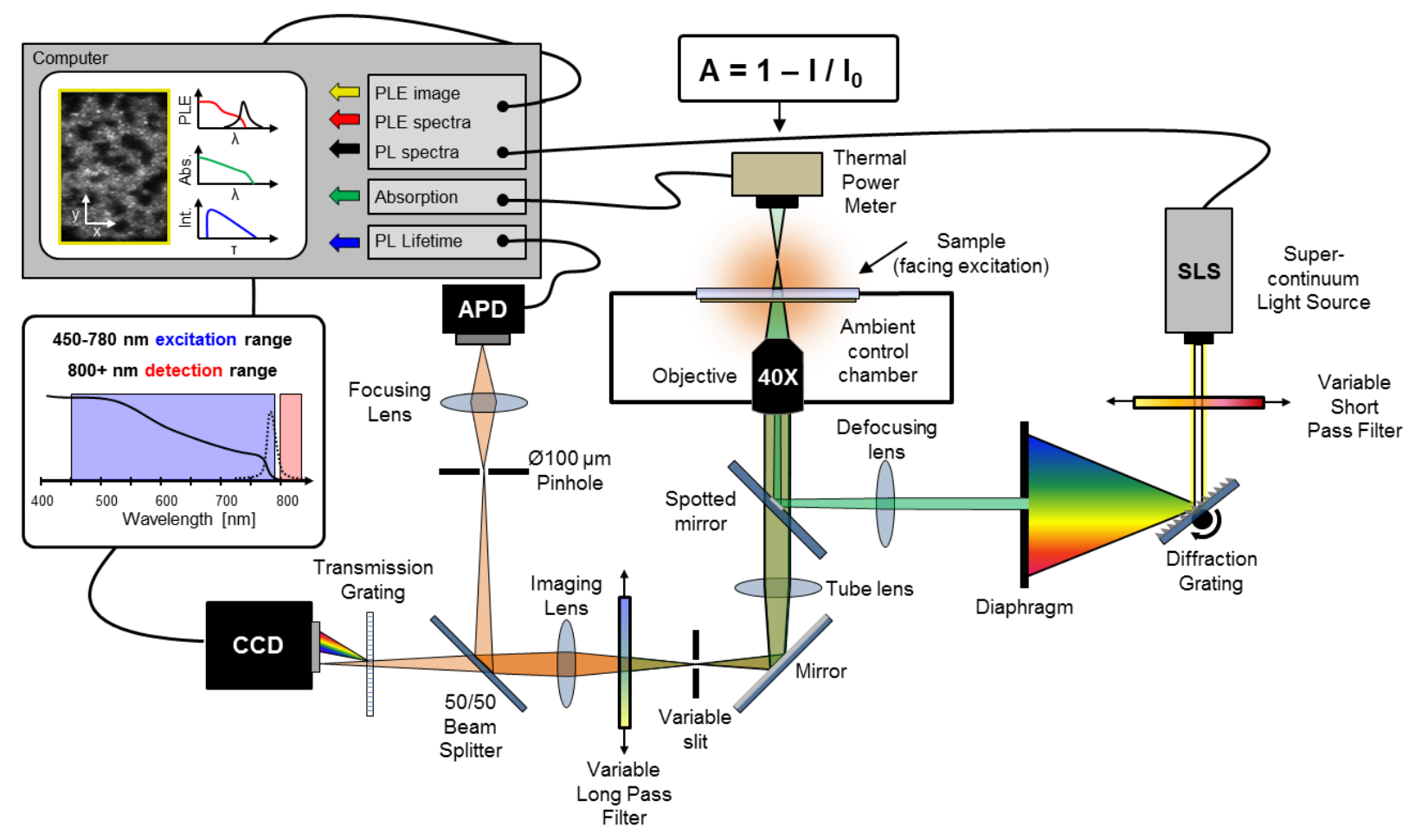

Figure S3. PL excitation/emission microscopy setup.

White laser light is generated by a supercontinuum light source (SLS, SuperK Extreme, NKT Photonics) and passing it via a diffraction grating monochromator provides the microscope with a tunable narrowband $(<1 \mathrm{~nm})$ excitation from $450 \mathrm{~nm}$ to $800 \mathrm{~nm}$ in $5 \mathrm{~nm}$ steps. The laser is pulsed (ca. 100 ps pulse width) allowing for timeresolved PL measurements using time correlated single photon counting when operating at $2 \mathrm{MHz}$ repetition rate as well as quasi steady-state PL measurements when operating at $80 \mathrm{MHz}$. The inverted epi-fluorescence wide-field fluorescence microscope based on the Olympus IX-71 model is equipped with a 40X (NA $=0.6$, Olympus LucPLANFl) long working distance objective, used for both excitation of the sample and collection of the photoluminescence (PL). The objective, together with the sample, resides in closed ambient control chamber (can be filled with nitrogen) in which the substrate covered with the studied film is placed so that sample faces the objective.

Above the sample we place a thermal power meter in order to measure the intensity of the laser excitation light transmitted by the sample $(\mathrm{I}(\lambda))$. By measuring the excitation power for each wavelength with an empty substrate $\left(I_{0}(\lambda)\right)$, we can calculate the absorptance by the sample as $A(\lambda)=1-I(\lambda) / I_{0}(\lambda)$.

The excitation beam is defocused in the sample plane to a spot of $50 \mu \mathrm{m}$ in diameter which generates a widefield PL image that is captured by the CCD camera (Princeton ProEM) with diffraction-limited resolution. One pixel in the image corresponds to $200 \mathrm{~nm}$ x $200 \mathrm{~nm}$ area on the sample. Prior to reaching the camera, the excitation is filtered out by two long pass filters working in conjunction to suppress the excitation and pass the PL. By tracking the intensity in the PL image for each excitation wavelength, we can construct the PL excitation spectrum 
which we can either average over the whole excited area of the sample or study in each single pixel of the image. Note that the diffraction limit at the emission wavelength doesn't allow resolution higher than $\sim 1 \mu \mathrm{m}$.

Since the excitation intensity is orders of magnitude higher than the PL intensity, the signal measured by the thermal meter is minimally influenced by the PL. In a complete excitation scan, we run the excitation from $450 \mathrm{~nm}$ to $800 \mathrm{~nm}$, which also allows us to measure absorption in the same wavelength range. The long pass filters for the emission are in this case adjusted such that only the tail of the $\mathrm{MAPbI}_{3}$ emission passes through (edge at $790 \mathrm{~nm}$ $+/-5 \mathrm{~nm}$ ) so that we can scan the excitation as close to the band edge as possible (see inset on the left in Figure S3). As such, PLE spectra are only acquired up to $780 \mathrm{~nm}$ while absorption spectra to $800 \mathrm{~nm}$. The reason we measure absorption at $800 \mathrm{~nm}$ is to determine the background level caused by light scattering that may be induced by morphological inhomogeneity typically seen in perovskites materials. ${ }^{1}$

\section{PLE and TRPL Excitation Conditions \& Calibration}

Operating at the highest repetition rate $(80 \mathrm{MHz})$, we assume a quasi-CW excitation condition since the carrier lifetime is longer than several tens of ns (see Figure 3). We calibrate the power for each excitation wavelength $\left(\lambda_{\mathrm{ex}}\right)$ to keep a constant photon flux $\left(2.3 \times 10^{17}\right.$ photons $\left.\times \mathrm{cm}^{-2} \times \mathrm{s}^{-1}\right)$, which effectively yields an excitation power density of $0.1 \mathrm{~W} / \mathrm{cm}^{2}$ at $\lambda_{\mathrm{ex}}=450 \mathrm{~nm}$, which reduces by a factor $\lambda_{\mathrm{ex}} / 450 \mathrm{~nm}$ for longer excitation wavelengths. When continuously scanning the excitation to produce the PLE spectra, we are simultaneously light-soaking the film and gradually degrading it. Thus, the excitation is used to both induce the $\mathrm{PbI}_{2}$ formation, detect its presence, and also to probe its influence on the recombination kinetics. We neglect changes in spectral features due to photon recycling since we collect emission from the low-energy tail of the emission, which is minimally affected by reabsorption.

TRPL was measured with excitation wavelengths varied in the range between $450 \mathrm{~nm}-700 \mathrm{~nm}$ in steps of $10 \mathrm{~nm}$ with a long pass filter at $720 \mathrm{~nm}$ in detection. The repetition rate was reduced to $2 \mathrm{MHz}$ while keeping the average power the same as in the PLE measurement, which yielded a pulse fluence of $50 \mathrm{~nJ} / \mathrm{cm}^{2}$ and $33 \mathrm{~nJ} / \mathrm{cm}^{2}$ for $\lambda_{\text {ex }}=450$ $\mathrm{nm}$ and $\lambda_{\mathrm{ex}}=700 \mathrm{~nm}$ respectively. For a film of thickness $\mathrm{d} \approx 400 \mathrm{~nm}$ (see SEM in Figure S1a), we estimate a photo-generated charge-carrier density of $\Delta \mathrm{n}=2.7 \times 10^{15} \mathrm{~cm}^{-3}$ at $\lambda_{\text {ex }}=450 \mathrm{~nm}$, and $\Delta \mathrm{n}=8 \times 10^{14} \mathrm{~cm}^{-3}$ at $\lambda_{\text {ex }}=700$ $\mathrm{nm}$ assuming the respective absorption coefficients $\alpha_{450}=1.2 \times 10^{5} \mathrm{~cm}^{-1}$ and $\alpha_{700}=3.3 \times 10^{4} \mathrm{~cm}^{-1}{ }^{2}$

\section{Extracting Spatially Resolved Spectra}

As demonstrated in Figure S1, the microscope forms an image at the position where the variable slit is placed. Thus, by narrowing the slit, we only allow a very small vertical segment of the image to be projected onto the CCD via the imaging lens. Prior to the camera, we place a transmission grating which is aligned such that both $0^{\text {th }}$ and $1^{\text {st }}$ diffraction orders arrive at the CCD chip. Thus, we retain the image of the narrow segment in the $0^{\text {th }}$ order (although at a reduced intensity) and acquire a spectrum of every point along the segment in the $1^{\text {st }}$ diffraction order. 


\section{SI Note 3. Stretched Exponential Fitting}

We apply a stretched exponential fit to all PL decays according to

$$
I(t)=A \exp \left[-\left(\frac{t}{\tau_{s t r}}\right)^{\beta}\right]
$$

where $\mathrm{A}$ is the amplitude, $\tau_{\text {str }}$ the effective lifetime and $\beta$ the dispersion coefficient. A process appears as a stretched exponential function when the rate becomes slower with time (for $0<\beta<1$ ).

The decay can be characterized also by the average lifetime, $<\tau>$ :

$$
\langle\tau\rangle=\frac{\tau_{s t r}}{\beta} \Gamma\left(\frac{1}{\beta}\right)
$$

where $\Gamma$ is the gamma function.

\section{Figure S4: Precursor modification and PLE/Absorptance}

We varied the precursor ratio according to the inset in Figure $\mathrm{S} 4 \mathrm{a}$ to produce an excess/lack of $\mathrm{PbI}_{2}$ in the $\mathrm{MAPbI}_{3}$ film and measured the PLE and absorptance spectra (Figure S4a,b). We see from the PLQY that the sample containing an excess $\mathrm{PbI}_{2}$ has a significantly higher PLQY than the others.
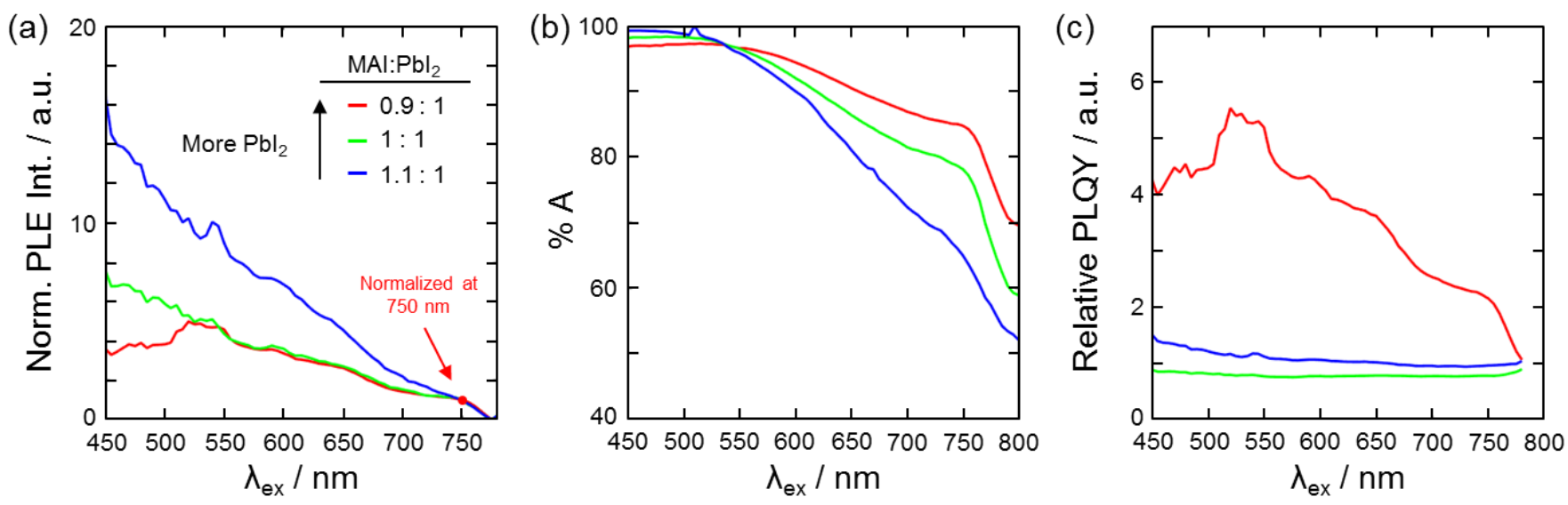

Figure S4. Precursor ratio modification shows difference in the (a) PLE, (b) absorptance and (c) PLQY spectra. 


\section{Figure S5: Measurements in Nitrogen vs. Humid Air}

We repeated the measurements demonstrated in Figure 2 in dry nitrogen and we did not observe any change in the absorption for nearly 1 hour of light-soaking. As soon as we opened the chamber and exposed the sample to humid air, the decomposition into $\mathrm{PbI}_{2}$ rapidly occurred (Figure S5a). For this experiment we increased the excitation power by 1 order of magnitude (10 Sun) in order to speed up the process since the point was to demonstrate the difference between the different ambient conditions. In Figure S5b we plot the average PLE spectra extracted from the scans in both ambient conditions and show in Figure S5c how the PLQY evolves for the three spectral ranges indicated with colored regions in Figure S5b.
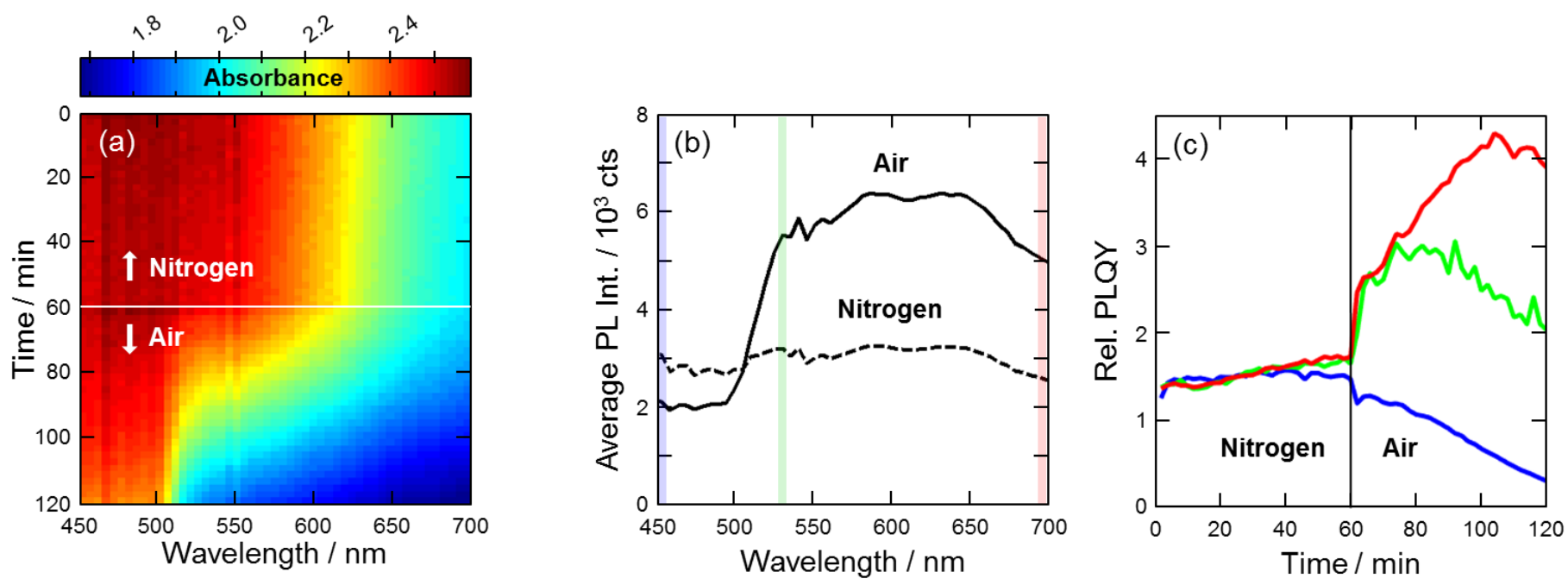

Figure S5. (a) 2D Map of the absorbance (note that it is not absorptance as in the main text) for excitation scans made in dry nitrogen for the first hour and in humid air for the second hour. (b) PLE spectra averaged over time in nitrogen (dashed trace) and air (solid trace). (c) PL intensity traces in time for three excitation wavelengths at $450 \mathrm{~nm}$ (blue trace), $530 \mathrm{~nm}$ (green trace) and $700 \mathrm{~nm}$ (red trace), which are also indicated with shaded regions in (b) with respective color. 


\section{Figure S6: Light soaking PLQY without Normalization}

Figure S6 shows the data from Figure 2 without normalization of the PLQY (Figure S6b). The overall PLQY increases steadily for nearly 4 hours of light soaking. Moreover, the increasing PLQY shows a clear dependence on the excitation wavelength (Figure S6c) where the appearance of a 'dip' emerges in the spectral range that coincides with $\mathrm{PbI}_{2}$ absorption $(450 \mathrm{~nm}-520 \mathrm{~nm})$. Thus, even if overall there are fewer photons absorbed due to the conversion from $\mathrm{MAPbI}_{3}$ to $\mathrm{PbI}_{2}$, there is a considerable improvement in the PLQY for wavelengths that are exclusively absorbed by the perovskite. Furthermore, we observe that for a significant amount of time (several hours of light soaking), the PLQY improves even for excitations between $450 \mathrm{~nm}-520 \mathrm{~nm}$ while presumably $\mathrm{PbI}_{2}$ is forming, which again brings up the question of the role $\mathrm{PbI}_{2}$ plays on the kinetics. In light of the different reports on whether $\mathrm{PbI}_{2}$ is good or bad (based on relative PLQY), it could be feasible that a different conclusion is reached depending on the initial concentration of residual $\mathrm{PbI}_{2}$ as well as how much $\mathrm{PbI}_{2}$ has formed (i.e. its coverage) by the time a measurement is made. ${ }^{3}$
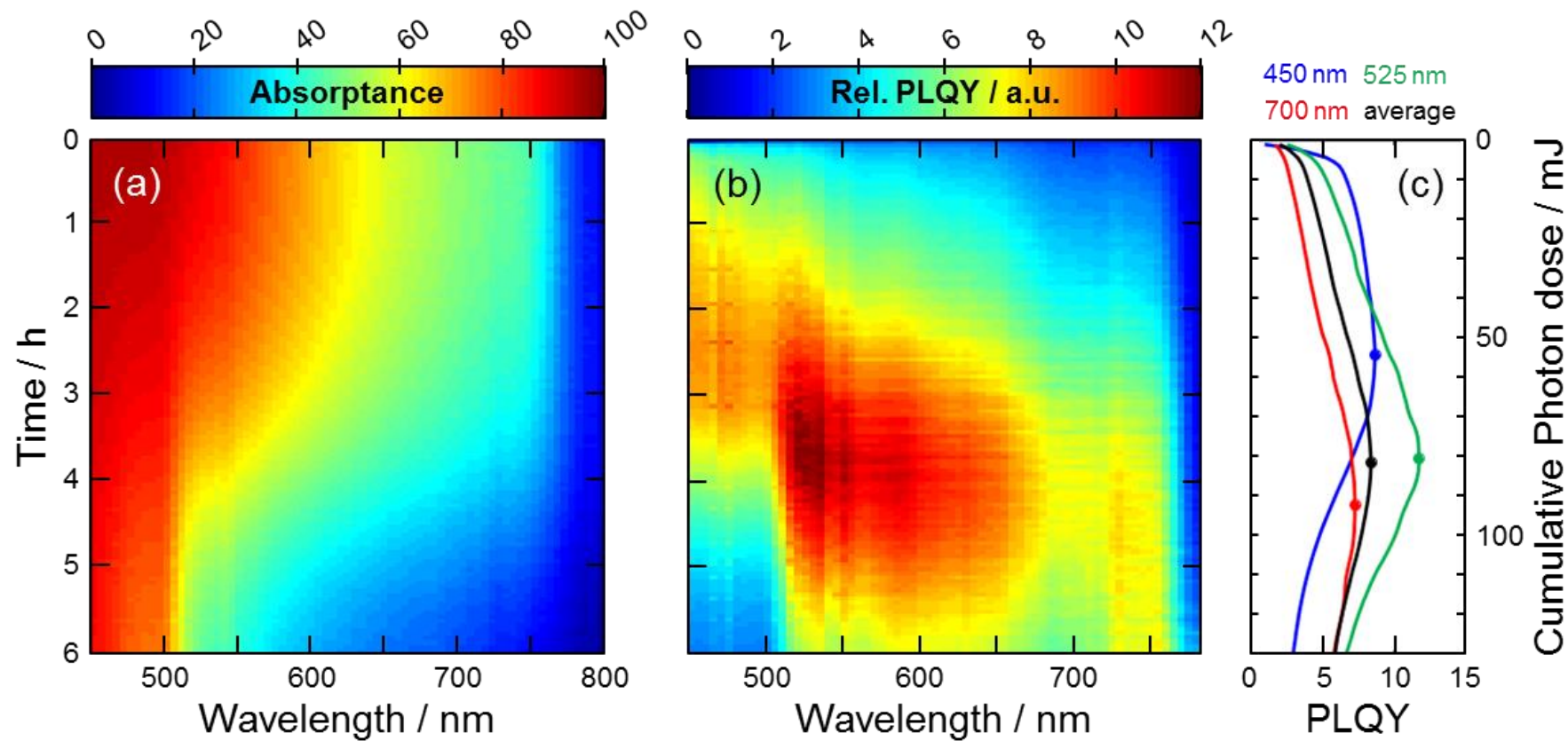

Figure S6. Data from Figure 2 without normalization showing (a) the absorptance and (b) the PLQY. (c) Evolution of the PLQY at three key wavelengths, $450 \mathrm{~nm}$ (blue trace), $525 \mathrm{~nm}$ (green trace), and $700 \mathrm{~nm}$ (red trace), as well as the spectrally averaged PLQY (black trace). 


\section{Figure S7: Complete TRPL Data Set with $<\tau>$ and $\beta$}

Figure S7a shows the normalized decays acquired with each of the 26 excitation wavelengths between $450 \mathrm{~nm}-$ $700 \mathrm{~nm}$. Figure S4b shows the PLQY without normalization where the PL enhancement can be observed. Figure S7c shows the average lifetime, $\langle\tau\rangle$, for each excitation wavelength and how it evolves throughout the 10-hour light-soaking experiment.

(a)

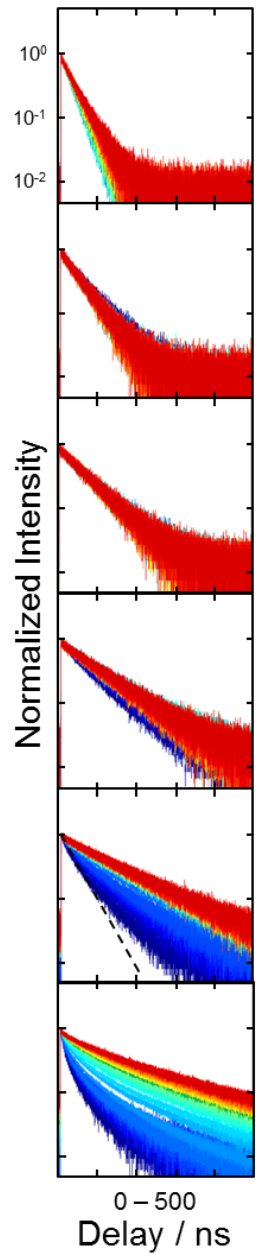

(b)

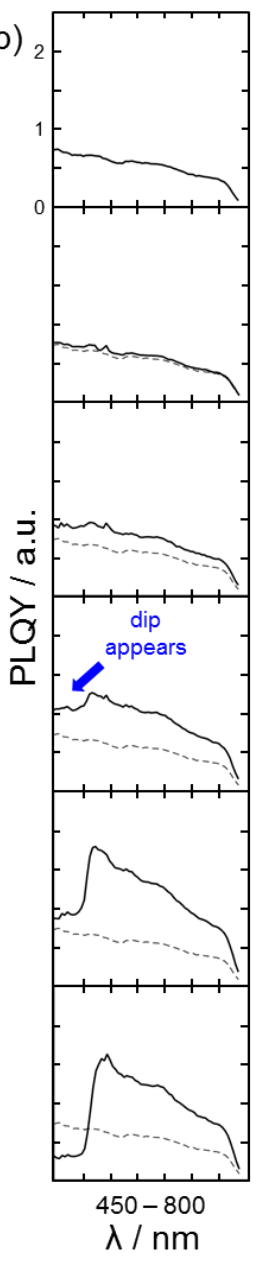

(c)

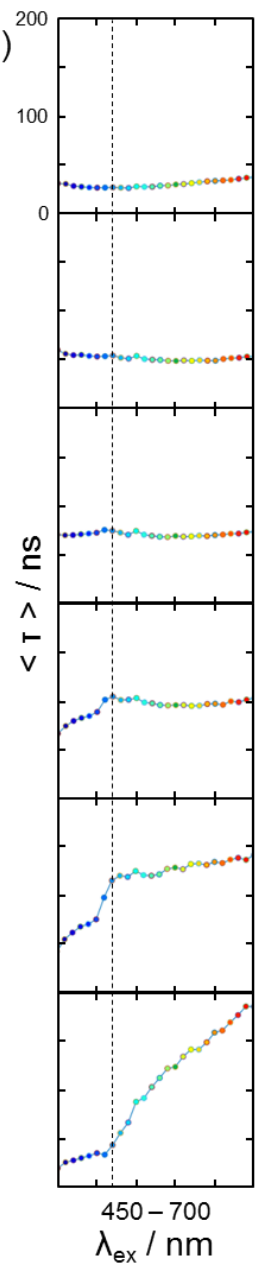

(d)

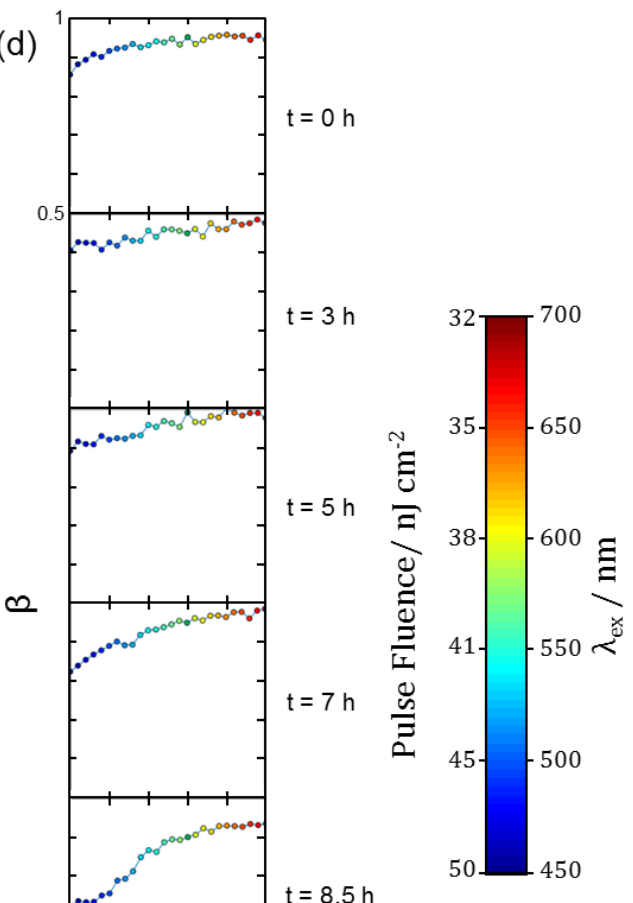

Figure S7. (a) Normalized PL decays for all excitation wavelengths. The colorbar on the right indicates the pulse fluence for each excitation wavelength. (b) The PLQY spectra from Figure 3a without normalization where the enhancement can be observed. (c) $<\tau>$ extracted for each wavelength (stretched exponential fitting). The vertical dashed line indicates the absorption onset for $\mathrm{PbI}_{2}$ where one can observe a drastic change in the lifetime once the signature of $\mathrm{PbI}_{2}$ appears in the PLQY spectrum in (b). (d) Beta values (stretched exponential fitting) extracted from the fit of all decays (see SI Note 3). 


\section{Figure S8: Fluence Dependence of PL Decays}

We verify that the fluence dependent fast component arises when charge carriers are generated directly in $\mathrm{PbI}_{2}$ by exciting the reference $\mathrm{MAPbI}_{3}$ sample through the substrate side (see schematics) and show that both $450 \mathrm{~nm}$ and $700 \mathrm{~nm}$ generate mono-exponential decays.
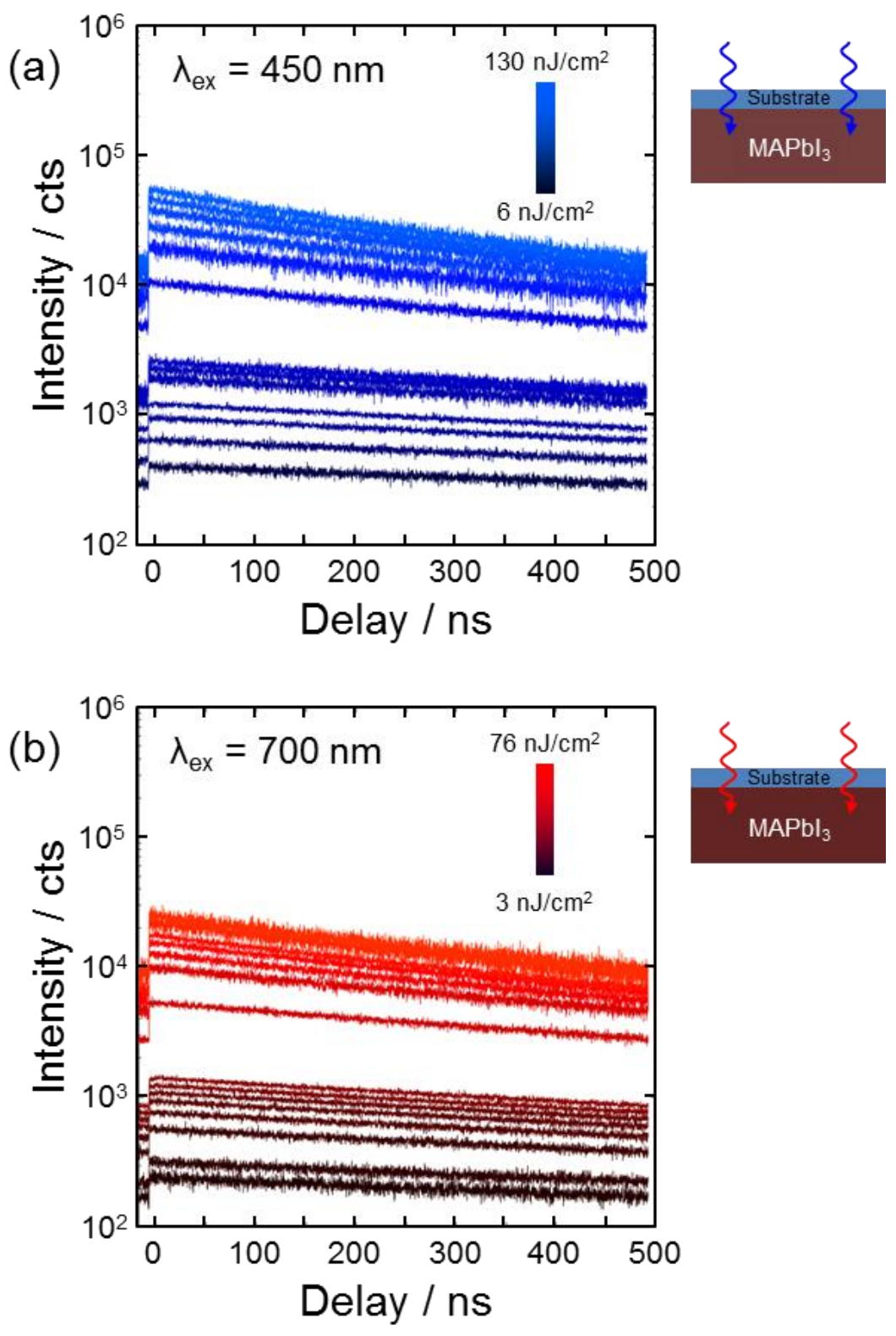

Figure S8. Fluence dependent PL decays generated with (a) $450 \mathrm{~nm}$ and (b) $700 \mathrm{~nm}$ excitation wavelengths measured through the substrate side of the reference $\mathrm{MAPbI}_{3}$ sample according to the schematics. PL is detected from the same side as the excitation. 


\section{Figure S9: Fluence Dependence of PL Emission}

We measured the fluence dependent PL spectra for $450 \mathrm{~nm}$ and $600 \mathrm{~nm}$ excitation in the same way as for Figure 4f, but measuring through the substrate side of the reference $\mathrm{MAPbI}_{3}$ sample. Figure $\mathrm{S} 9$ shows that PL emission with both excitation wavelengths over the entire fluence range generates the same PL emission with a peak at $775 \mathrm{~nm}$. Thus, we verify that the blue-shifted PL is generated when charge carriers are generated directly in $\mathrm{PbI}_{2}$

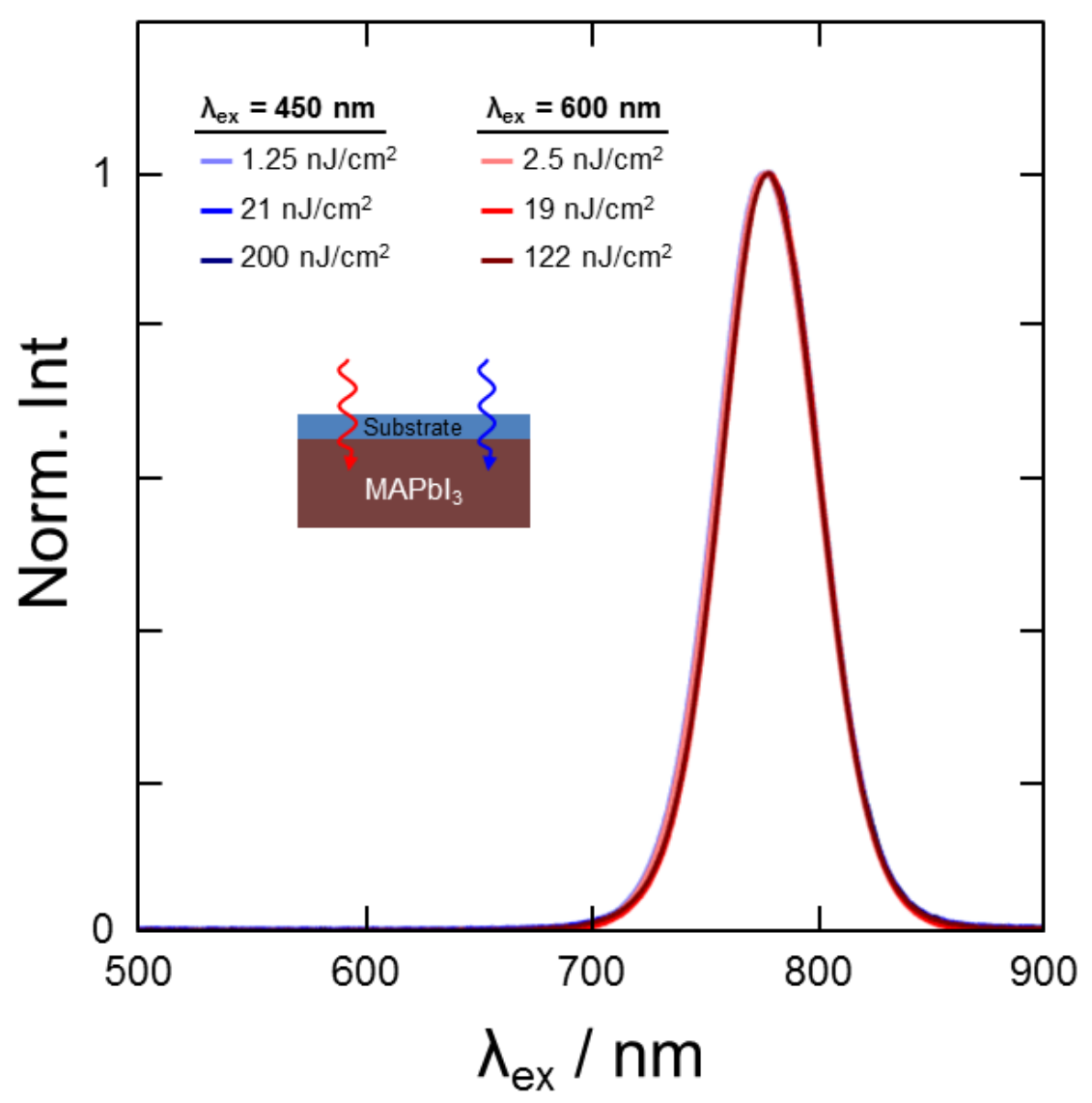

Figure S9. Fluence dependent PL spectra generated with (a) $450 \mathrm{~nm}$ and (b) $700 \mathrm{~nm}$ excitation wavelengths measured through the substrate side of the reference $\mathrm{MAPbI}_{3}$ sample according to the schematic. PL is detected from the same side as the excitation. 


\section{Figure S10-11: PL images acquired with different excitation wavelengths}

Using a long-pass filter at $610 \mathrm{~nm}$, we generate PL images with different excitation wavelengths ranging from $450 \mathrm{~nm}$ to $600 \mathrm{~nm}$ to demonstrate the presence of emitting domain when exciting in the range between $450 \mathrm{~nm}-$ $520 \mathrm{~nm}$ (clearly visible only to about $500 \mathrm{~nm}$ ). In the series of images shown in Figure S10-S11, we divide all images with the image acquired at $600 \mathrm{~nm}$. The reason we do this is to better demonstrate that the domains disappear as the distribution of PL becomes more and more homogeneous as the excitation wavelength approaches $600 \mathrm{~nm}$. In Figure $\mathrm{S} 10$ we excite the sample through the $\mathrm{PbI}_{2}$ layer first where the domains are clearly visible. In Figure $\mathrm{S} 11$ we flip the sample upside down and excite directly into $\mathrm{MAPbI}_{3}$ where we do not observe any difference in the series of images.
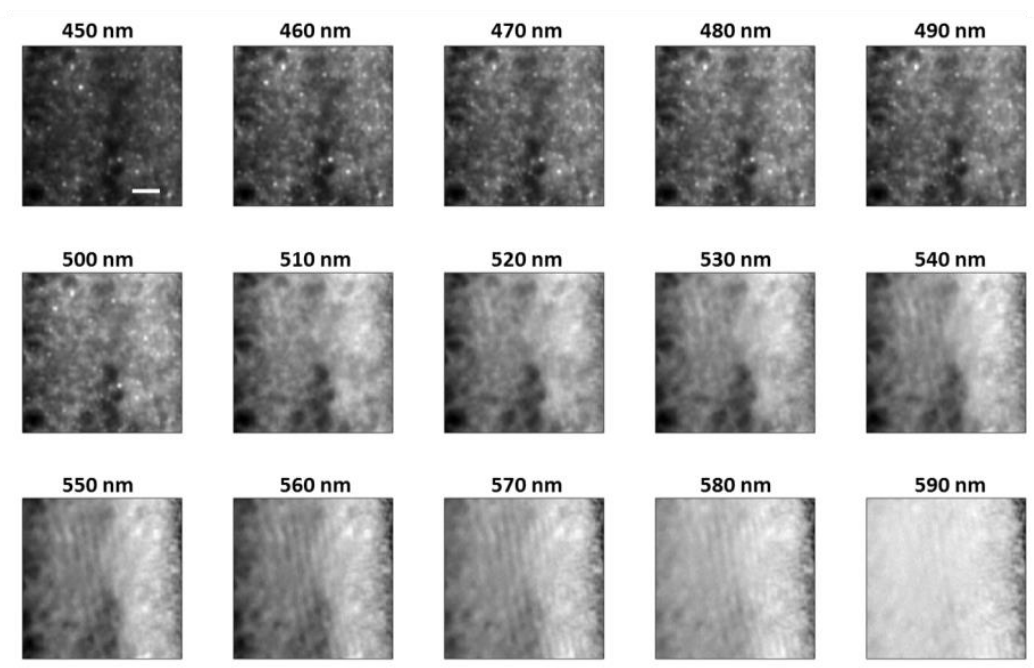

Figure S10. Series of images acquired with different excitation wavelengths (indicated at top of each image) which have been normalized to the image acquired at $600 \mathrm{~nm}$. The measurement geometry is such that the excitation light is incident on the $\mathrm{PbI}_{2}$ layer first, from which we also acquire the PL image. The domains are clearly visible between $450 \mathrm{~nm}-500 \mathrm{~nm}$, after which their intensities start to diminish. The scale bar represents $5 \mu \mathrm{m}$.
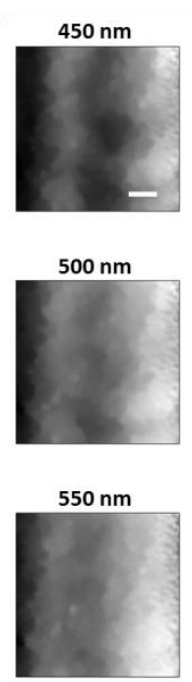
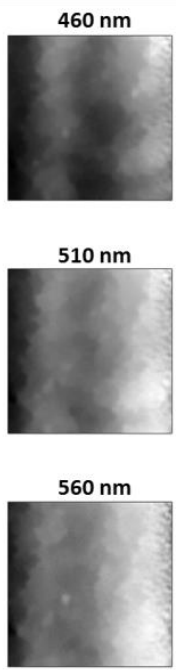
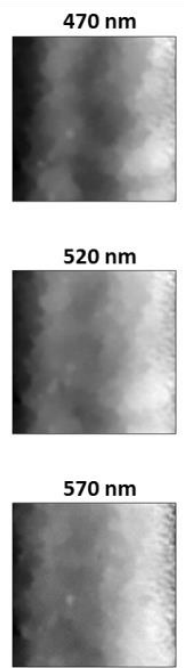
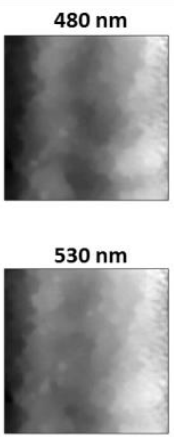

$580 \mathrm{~nm}$

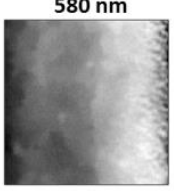

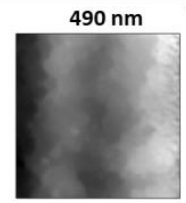

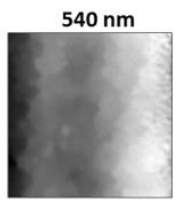

$590 \mathrm{~nm}$

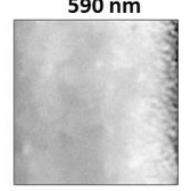

Figure S11. Series of images acquired with different excitation wavelengths (indicated at top of each image) which have been normalized to the image acquired at $600 \mathrm{~nm}$. The measurement geometry is such that the excitation light is incident on the MAPbI 3 layer first, from which we also acquire the PL image. No domains are seen and the distribution of PL appears rather homogeneous for all excitation wavelengths. The scale bar represents $5 \mu \mathrm{m}$. 


\section{References}

(1) Tian, Y.; Scheblykin, I. Artifacts in Absorption Measurements of Organometal Halide Perovskite Materials: What Are the Real Spectra?. J. Phys. Chem. Lett. 2015, 6, 3466-3470.

(2) Green, M.A.; Jiang, Y.; Soufiani, A.M.; Ho-Baillie, A.; Optical Properties of Photovoltaic OrganicInorganic Lead Halide Perovskites. J. Phys. Chem. Lett. 2015, 6, 4774-4785.

(3) Jacobsson, T. J.; Correa-Baena, J. P.; Halvani Anaraki, E.; Philippe, B.; Stranks, S. D.; Bouduban, M. E. F.; Tress, W.; Schenk, K.; Teuscher, J.; Moser, J. E.; et. al. Unreacted PbI 2 as a Double-Edged Sword for Enhancing the Performance of Perovskite Solar Cells. J. Am. Chem. Soc. 2016, 138, 10331-10343. 